\title{
Versatile salts as a strategy to modify the biopharmaceutical properties of venlafaxine and potential hypoglycemic effect study
}

Ningbo Gong ${ }^{1,2}$, Yong Zhang ${ }^{2}$, Ying Wang ${ }^{1}$, Hongmei Yu${ }^{1}$, Baoxi Zhang ${ }^{1}$, Hailu Zhang ${ }^{3, *}$, Yang $\mathrm{Lu}^{1, *}$, Guanhua $\mathrm{Du}^{4}$

Table S1 The ionic bonds and hydrogen-bond geometry (in $\AA$ and deg)

\begin{tabular}{|c|c|c|c|c|c|c|}
\hline Form & 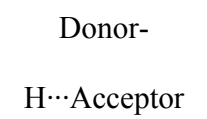 & $\mathrm{D}-\mathrm{H}(\AA)$ & $\begin{array}{l}\mathrm{H} \cdots \mathrm{A} \\
(\AA)\end{array}$ & $\begin{array}{l}\mathrm{D} \cdots \mathrm{A} \\
(\AA)\end{array}$ & $\begin{array}{c}\angle \mathrm{DHA} \\
\left(^{\circ}\right)\end{array}$ & symmetry code \\
\hline \multirow{3}{*}{ VLF : MA } & N1-H1_..O4 & 0.966 & 1.767 & 2.684 & 157.2 & $\mathrm{x}, \mathrm{y}, \mathrm{z}+1$ \\
\hline & O6-H6A...O5 & 0.820 & 1.613 & 2.418 & 166.7 & $\mathrm{x}, \mathrm{y}, \mathrm{z}$ \\
\hline & $\mathrm{O} 2-\mathrm{H} 2 \ldots \mathrm{O} 5$ & 0.820 & 2.090 & 2.874 & 160.8 & $x, y, z$ \\
\hline \multirow{3}{*}{ VLF : PA } & N1-H1_..O6 & 0.980 & 1.802 & 2.693 & 149.6 & $\mathrm{x}, \mathrm{y}+1, \mathrm{z}$ \\
\hline & $\mathrm{O} 2-\mathrm{H} 2 \cdots \mathrm{O} 3$ & 0.820 & 2.030 & 2.826 & 163.79 & \\
\hline & $\mathrm{O} 3-\mathrm{H} 3 \cdots \mathrm{O} 5$ & 0.820 & 1.788 & 2.594 & 167.01 & \\
\hline \multirow{2}{*}{ VLF : 4-CA } & N1-H1_..O4 & 0.980 & 1.612 & 2.565 & 163.2 & $-x+1 / 2, y+1 / 2, z-1 / 2$ \\
\hline & $\mathrm{O} 2-\mathrm{H} 2 \mathrm{~A} \cdots \mathrm{O} 3$ & 0.820 & 1.932 & 2.750 & 175.77 & $-x+1 / 2, y+1 / 2, z-1 / 2$ \\
\hline
\end{tabular}

Table S2 The main powder XRD peaks of salts and their raw materials

\begin{tabular}{lll}
\hline No & sample & \multicolumn{1}{c}{ PXRD peak } \\
\hline 1 & VLF & $8.08^{\circ}, 11.06,15.74^{\circ}, 16.24^{\circ}, 16.80^{\circ}, 19.98^{\circ}, 21.48^{\circ}, 22.54^{\circ}, 26.16^{\circ}$ \\
2 & PA & $15.36^{\circ}, 18.54^{\circ}, 21.16^{\circ}, 22.20^{\circ}, 26.98^{\circ}, 30.53^{\circ}, 37.64^{\circ}$ \\
3 & VLF : PA & $7.02^{\circ}, 14.06^{\circ}, 15.34^{\circ}, 17.76^{\circ}, 22.28^{\circ}, 25.33^{\circ}, 25.70^{\circ}, 26.38^{\circ}$ \\
4 & 4-CA & $18.48^{\circ}, 24.52^{\circ}, 25.18^{\circ}, 27.64^{\circ}, 28.34^{\circ}, 29.72^{\circ}$ \\
5 & VLF : 4-CA & $13.48^{\circ}, 15.44^{\circ}, 15.96^{\circ}, 16.70^{\circ}, 18.78^{\circ}, 20.04^{\circ}, 21.70^{\circ}, 23.32^{\circ}$, \\
& & $26.72^{\circ}, 31.26^{\circ}$ \\
6 & 4-HA & $17.54^{\circ}, 19.42^{\circ}, 22.22^{\circ}, 24.44^{\circ}, 26.76^{\circ}, 29.84^{\circ}$ \\
7 & VLF : 4-HA & $8.62^{\circ}, 12.62^{\circ}, 14.94^{\circ}, 16.04^{\circ}, 17.30^{\circ}, 17.86^{\circ}, 18.60^{\circ}, 19.24^{\circ}$, \\
& & $20.40^{\circ}, 21.76^{\circ}, 22.92^{\circ}, 24.50^{\circ}$ \\
8 & SA & $12.70^{\circ}, 18.08^{\circ}, 23.64^{\circ}, 24.38^{\circ}, 25.58^{\circ}, 27.38^{\circ}, 34.18^{\circ}$ \\
9 & VLF : SA & $11.02^{\circ}, 11.28^{\circ}, 14.16^{\circ}, 15.38^{\circ}, 18.34^{\circ}, 19.98^{\circ}, 20.28^{\circ}, 21.58^{\circ}$, \\
& & $22.00^{\circ}, 22.80^{\circ}, 23.06^{\circ}, 23.74^{\circ}$ \\
\hline
\end{tabular}


Fig S1 The TGA patterns of VLF salts
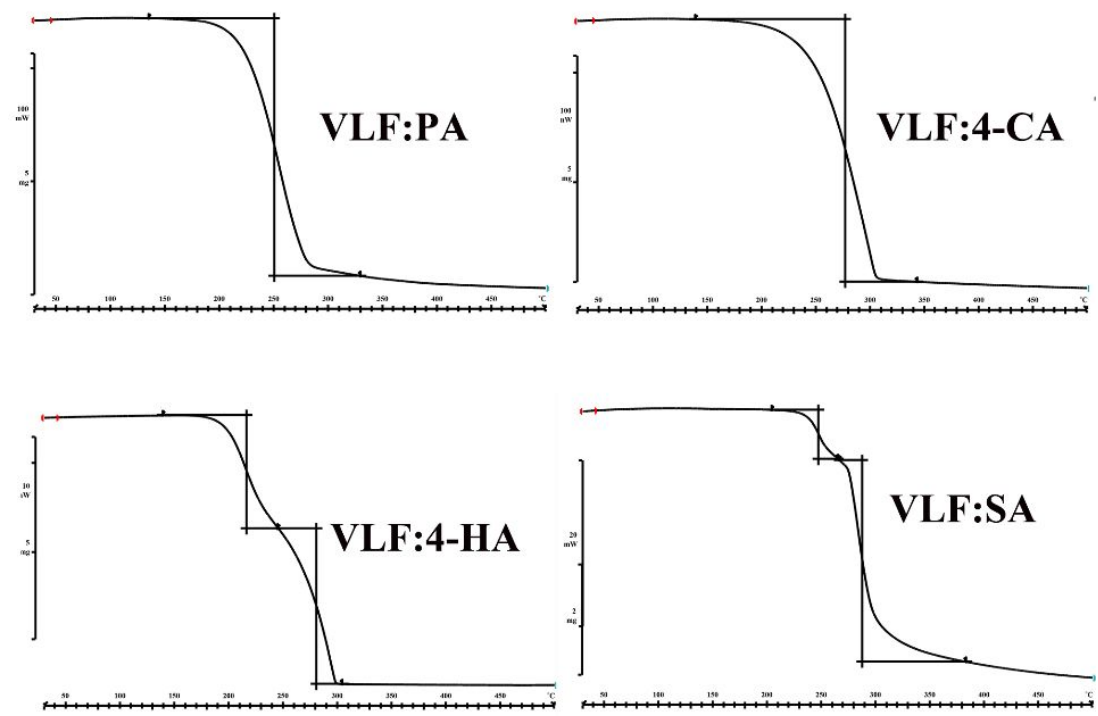

Fig S2 The dissolution profiles of VLF salts in pure water.

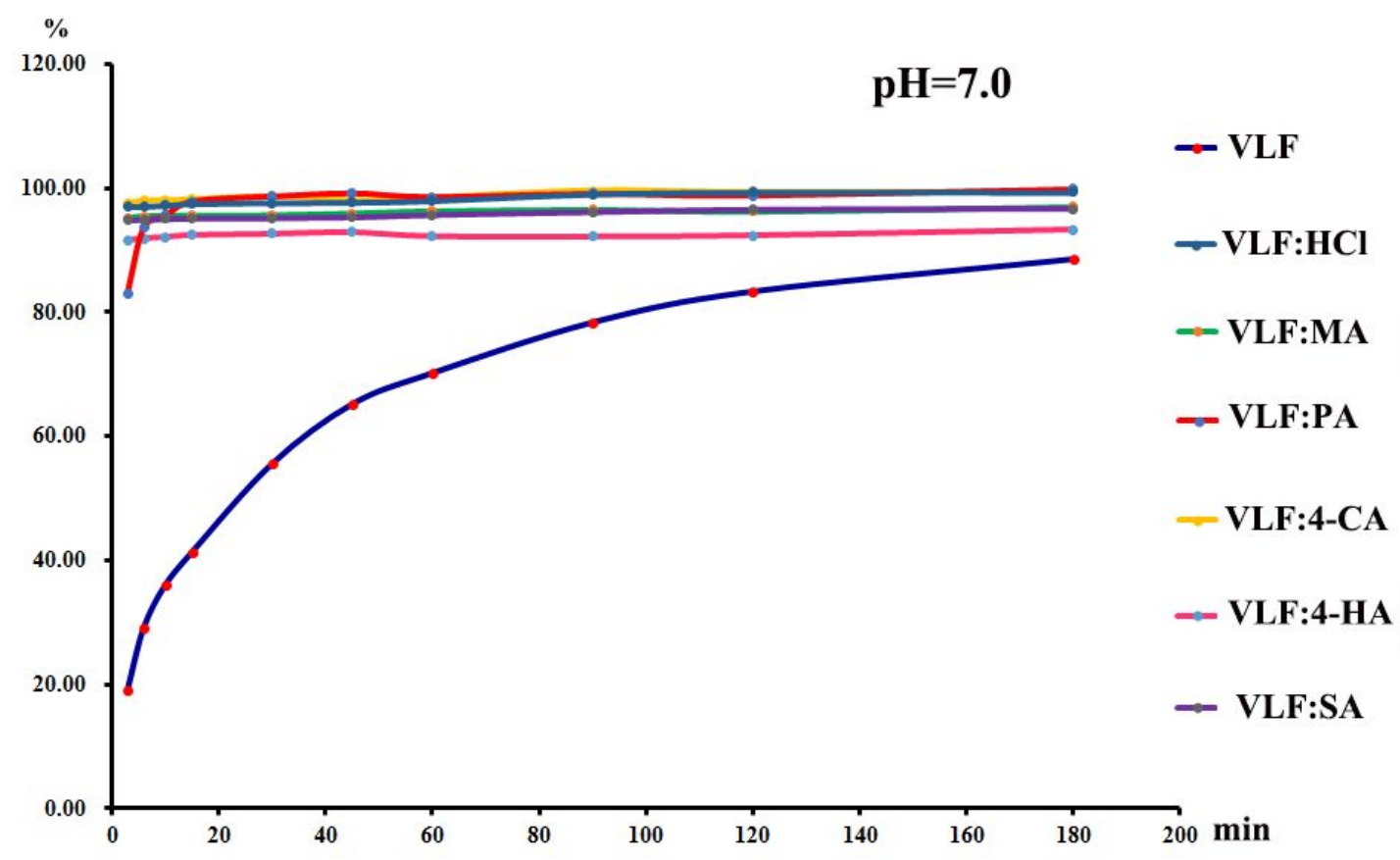

